\title{
International myeloma working group consensus recommendations on imaging in monoclonal plasma cell disorders
}

\begin{abstract}
Recent advances in the treatment of multiple myeloma (MM) have increased the need for accurate diagnosis of the disease. The detection of bone and bone marrow lesions is crucial in the work-up of MM, and often dictates the decision to start treatment. Furthermore, detection of minimal residual disease (MRD) is important for prognosis and treatment planning, and has underscored an unmet need for sensitive imaging modalities that accurately assess response to therapy in MM. Low dose whole body computed tomography (WBCT) has increased sensitivity compared to conventional skeletal survey (CSS) in the detection of bone disease, and can reveal information leading to changes in therapy and management that could prevent or delay the onset of significant morbidity and mortality related to skeletal-related events. Given the multiple options for detection of bone and bone marrow lesions ranging from CSS to WBCT, positron emission tomography (PET)-CT, and magnetic resonance imaging (MRI), the International Myeloma Working Group has established guidelines on the optimal and standardized use of imaging modalities in different stages of the disease. These recommendations on imaging within and outside of clinical trials will help to standardize the imaging worldwide in order to allow comparison of results and unification of treatment approaches.
\end{abstract}

\section{INTRODUCTION}

MM is caused by the infiltration and proliferation of malignant monoclonal plasma cells, primarily in the bone marrow. There is evidence that it is always preceded by precursor stages of monoclonal gammopathy of undetermined significance (MGUS) and smoldering multiple myeloma (SMM) (1). The early stages of the disease are defined by the presence of monoclonal plasma cells in the bone marrow-with $10 \%$ or more being the defining percentage for SMM compared to MGUS-along with the presence of monoclonal protein in serum and/ or urine (2). Sometimes, MM can be preceded by a solitary accumulation of clonal plasma cells either in the bone or the soft tissue (solitary plasmacytoma or SPC) without signs of systemic disease (2).

Usually, the diagnosis of a monoclonal plasma cell disorder is made based on immunofixation electrophoresis on serum and urine samples and on bone marrow biopsies (3). The main presenting symptoms of MM have been given the acronym 'CRAB', and include hypercalcemia $(C)$ and bone destruction $(B)$ due to an over-activation of osteoclasts, renal impairment mostly caused by monoclonal light chains affecting the kidneys $(R)$, and anemia (A) reflecting amongst other things infiltration of the bone marrow with replacement of the physiological hematopoiesis (2). Given that $80-90 \%$ of all MM patients develop bone disease (4), a thorough assessment of the degree of skeletal involvement and damage to structural integrity is of utmost importance. Furthermore, whole body imaging techniques have revealed that MM does not always affect mineralized bone and bone marrow in a homogeneous way. In fact, approximately $60 \%$ of patients have plasma cell accumulation and bone destruction occurring in a focal or patchy fashion without osteolysis. These lesions are referred to as focal lesions, and are different from lytic lesions where bone destruction has already taken place (5).

The clinical use of imaging modalities to diagnose MM is oftentimes influenced by availability and affordability of different techniques rather than by scientific data alone. Therefore, the present guidelines aim to provide a rationale for the use of different imaging modalities at various time points along the continuum of plasma cell disorders, as well as to provide recommendations regarding imaging in specific clinical scenarios. These guidelines may also serve as the basis for further research questions. 


\section{General clarifications}

Due to a paucity of randomized data on MM imaging, this paper does not apply levels of evidence to the guideline recommendations. It is also important to highlight that treatment should be considered for any patient meeting the active MM criteria (2) even if imaging is negative. The same approach should hold true for MM in biochemical or clinical relapse/progression. In this manuscript, we will use the term 'Whole body MRI' (WBMRI), which should not be confused with a cancer screening technique e.g. for patients with cancer of unknown primary. Specific recommendations on how to perform this imaging technique have been accepted for publication (Messiou et al, accepted, Radiology 2018).

\section{Availability of Imaging Modalities}

Low dose whole body computed tomography (WBCT) is preferred over conventional skeletal survey (CSS) for the diagnosis of MM bone disease. However, the panel is aware that the novel imaging techniques mentioned in this paper are not available at all locations worldwide. We are also aware that in some countries, financial considerations may preclude even CSS in patients in whom MM is diagnosed based on other myeloma defining events (MDE). Therefore, despite the shortcomings mentioned in this manuscript, CSS can be utilizied when WBCT or other novel imaging methods are not available.

Although WBMRI is preferred in some situations, it is not available in many institutions in the world. MRI of the spine and pelvis is a reasonable alternative to provide sufficient bone marrow imaging. However, even this may be limited by the ability of patients to tolerate the procedure. In these situations, PET-CT or a more limited MRI assessment may need to be conducted.

PET-CT can be used in place of WBCT, but is dependent on availability. However, if a PETCT is used instead of a WBCT, it is necessary that the CT part of PET-CT fulfills the criteria of a diagnostic WBCT.

Finally, the panel recognized that limited imaging of symptomatic areas in MM are needed throughout the MM disease course and that the choice of the specific imaging modality is dependent on both the location of the symptom, and the area involved (e.g. bone versus extramedullary). Recommendations on such focused imaging are beyond the scope of this paper.

\section{Replacement of conventional skeletal survey by WBCT}

Historically, CSS has been applied for the assessment of MM bone disease (6) because of its low costs and widespread availability. Therefore, it has been broadly used and has formed the basis for previous guidelines and diagnostic models (7). However, this technique has significant limitations, particularly regarding sensitivity. As evidence of this, Edelstyn et al. showed in 1967 that up to $30-50 \%$ bone loss was required before it could be detected in conventional x-ray (8).

WBCT either alone or in combination with PET has been shown to provide significantly superior sensitivity for the detection of osteolytic lesions in MM patients. In a multi-center analysis, the International Myeloma Working Group (IMWG) compared CSS and WBCT scans from 212 patients with monoclonal plasma cell disorders, finding that WBCT was positive in $25.5 \%$ of patients who had negative CSS. This difference was mainly due to the superior detection rate in the spine and pelvis, because when comparing the sensitivity of CSS and WBCT in the long bones, no significant difference could be determined (9). Similar findings had been reported previously in a different series of 32 patients with $50 \%$ positive patients in X-ray and $74 \%$ positive in CT of spine and pelvis (10). In another study of 29 patients $5(17 \%)$ showed osteolytic lesions in WBCT despite CSS being negative (11). Finally, in a cohort of 52 patients in all stages of monoclonal plasma cell disorders, $12(23 \%)$ were positive by CT and negative by CSS, confirming the increased sensitivity of WBCT (12). 
In the adult long bones, MM infiltration can be detected by CT as nodular or even diffuse manifestations and appears to have prognostic significance as well as correlation with treatment response $(13,14)$. Additionally, WBCT compared to CSS is more comfortable for the patient since the study is performed in the supine position and has a short acquisition time.

\section{Technical considerations with WBCT}

To avoid missing osteolytic lesions in the humeri in WBCT, the arms should not be placed above the head but rather next to the body to keep them in the field of view, and on cushions to avoid weakening of the CT beam in the area of the thoracic and lumbar spine.

In general, it is recommended to perform a whole body low dose multi-detector CT; doses as low as 3.2-4.8 mSv have been reported to be sufficient. In comparison, radiation dose of CSS is usually 1.2-2.4 mSv (11,15-17). Recently, an expert group consisting of radiologists and hematologists has analyzed data on this topic to provide further recommendations(18).

\section{Bone marrow imaging}

The higher sensitivity of modern imaging techniques such as PET-CT and WBMRI provides the opportunity not only to determine bone destruction in MM but also to assess tumor burden and disease activity in a large area, if not the whole bone marrow compartment. As mentioned earlier, bone marrow infiltration in MM is not homogeneous in the majority of patients. Therefore, the ability to identify discrete areas of diffuse versus focal plasma cell infiltration by sensitive imaging techniques provides a novel dimension to disease burden and response assessment. Until now, in most cases at initial diagnosis, genetic testing for risk assessment and definition of complete remission $(\mathrm{CR})$ as well as minimal residual disease (MRD) assessment in MM rely on the plasma cell percentage and biology found in a bone marrow specimen taken essentially at random either from the iliac crest or the sternum. However, these sites may or may not be representative for the real disease burden. Given this caveat, it is noteworthy that a retrospective study by the Arkansas group has shown that in some but not all patients, genomic findings of MM cells from a random sample and an imaging guided biopsy of a focal lesion can in some cases be similar and in others different, respectively (19). In a prospective study, the group from Heidelberg has shown that also the plasma cell percentage differs significantly (20). Given this heterogeneity and its potential to impact clinical care decisions, more comprehensive bone marrow imaging is highly desirable in MM.

\section{Comparison of PET-CT and WBMRI for bone marrow imaging}

PET-CT and WBMRI provide different and complementary information on the investigated tissue. While WBMRI is based on examining the composition of the tissue regarding water and fat content, PET-CT draws information from metabolic activity of the cells within the investigated area, which are taking up a radioactive tracer.

In PET-CT the most widely used tracer is $18 \mathrm{~F}$ Fluorodeoxyglucose (FDG) with the radionuclide 18 Fluorine. If PET-CT imaging is mentioned in this manuscript, this refers to 18F FDG PETCT. FDG is ingested by cells in accordance with their glucose metabolism and therefore with their energy consumption.

In WBMRI contrast agents are usually based on gadolinium which has been shown to be relatively inert, but in the case of renal insufficiency can lead to a severe complication: namely, nephrogenic systemic fibrosis (21). However, the indications where a contrast agent is needed for WBMRI in MM patients are rare, as already conventional non-enhanced WBMRI has a high resolution and contrast with regards to the bone marrow.

While PET-CT is superior to MRI regarding the assessment of viability of focal lesions, a diffuse infiltration can be better assessed in MRI due to its higher spatial resolution.

Diffusion weighted imaging (DWI) is an MRI technique measuring the movement of water molecules in the tissue (22). At present, the DWI technique is not validated sufficiently to be recommended for general application. Similarly, data with the combined PET-MRI scanners and novel tracers are not mature for the purposes of this guideline paper $(23,24)$.

\section{RECOMMENDATIONS IN DIFFERENT STAGES OF THE DISEASE}




\section{Monoclonal gammopathy of undetermined significance}

MGUS has a high incidence of $3.2 \%$ in individuals of 50 years of age or older and of $5.3 \%$ in persons of 70 years or older (25). According to the recently updated analysis of the Southeastern Minnesota cohort, risk factors for MGUS to active MM progression include an M-protein of $1.5 \mathrm{~g} / \mathrm{dl}$ or more and an abnormal free light chain ratio in patients with non-lgM MGUS. Patients with no or 1 risk factor had a progression rate of $7 \%$ and $20 \%$ within 20 years respectively, while individuals with 2 risk factors showed a progression rate of $30 \%$ in 20 years (26). Therefore, the IMWG decided to recommend whole body imaging only in patients with high risk MGUS. Because the most important symptom to be excluded in patients with monoclonal gammopathy is bone destruction and due to the benefits of WBCT mentioned above, we recommend this technique as the primary modality to be used. Recommendations are summarized in Figure 1 and the grey box below. IgM MGUS usually does not develop into MM but Waldenstrom's Macroglobulinemia and no routine bone imaging is recommended for this entity.

MRI has been investigated in MGUS, resulting in recognition of focal lesions in different reported series from $3.5 \%$ to $23.4 \%(27-29)$. However, confirmative histological examination of the focal lesions identified was not performed in any of these studies, and there is a high probability of false positive findings. WBMRI can be useful in selected patients where there is concern for MM based on equivocal WBCT results. The prevalence of MGUS increases with age, and distinguishing any MM related bone changes from a benign etiology becomes very important. For example, age-related osteoporosis in elderly individuals is usually accompanied by a higher fat content in the bone marrow due to replacement of the physiological hematopoiesis by fat cells, whereas osteoporosis caused by MM goes along with a higher cellularity due to malignant infiltration. These changes can be teased out by the use of WBMRI, especially in case of vertebral fractures (30-32). Furthermore, presence or absence of bone marrow edema allows an assessment of the age of the fracture (new versus old). Oligosecretory disease occurs in about 3-5\% of MM patients (4) and remains in the differential diagnosis during evaluation of an MGUS patient. WBMRI can help identify this subset of patients as well. To date, there are no published data on PET-CT findings in MGUS patients.

\section{Recommendations for imaging in MGUS}

- In suspected high risk non IgM MGUS, we recommend WBCT to rule out MM. If WBCT is not available, conventional skeletal survey or WBMRI are alternatives.

- In patients with equivocal findings on WBCT (or skeletal survey) in whom there is concern for myeloma, we recommend WBMRI (or MRI of the spine and pelvis if WBMRI is not available).

We do not recommend follow up bone imaging unless there are signs of progression to symptomatic disease e.g. pain or increase in serological parameters.

\section{Solitary plasmacytoma}

SPC occurs as solitary bone (SBP) or extramedullary lesion (EMP), with the former being about twice as prevalent as the latter (33). Furthermore, SBP has a significantly higher risk of progression into MM compared to EMP, with $35 \%$ versus $7 \%$ within 2 years, respectively (33). The most important information acquired from whole body imaging in any patients with SPC is the exclusion of additional osteolytic lesions or further soft tissue masses, which would constitute systemic MM. WBMRI and PET-CT provide high sensitivity and specificity to detect both (further) diffuse as well as focal bone marrow infiltration. Different guidelines have preferred PET-CT over WBMRI of the spine and pelvis. However, since WBMRI has a higher 
sensitivity for the diffuse infiltration part of the bone marrow, WBMRI would be the first choice in patients with SBP. If a WBMRI is not available PET-CT provides a reasonable alternative. In EMP, PET-CT has been preferred to exclude further lesions.

Since the risk of progression of SPC into MM or relapse is relatively high with $14-38 \%$ within the first 3 years (34), yearly follow up with the same imaging technique used at first diagnosis should be performed for the first 5 years and later on only in case of clinical or laboratory signs or symptoms even though there is no clear evidence for the benefit of this procedure yet. Figure 2 and the respective grey box show the recommendations for imaging in patients with SPC.

\section{Recommendations for imaging in SPC}

- We recommended WBMRI (or MRI of the spine and pelvis, if WBMRI is not available) in patients with newly diagnosed solitary bone plasmacytoma.

- We recommend PET-CT in patients with newly diagnosed solitary extramedullary plasmacytoma.

- If WBMRI is not available, WBCT or PET-CT can be used as alternatives in patients with newly diagnosed solitary bone plasmacytoma.

- The same technique used at initial diagnosis should be repeated in yearly intervals for at least 5 years.

\section{Smoldering multiple myeloma}

Compared to active MM, the disease burden is relatively lower in SMM patients. This makes it very important to apply imaging techniques with a high sensitivity to identify bone disease/involvement and distinguish them from active MM. Several retrospective analysesboth with MRI and PET-CT-have been performed. In two independent datasets of 149 and 67 patients with SMM using WBMRI and spinal MRI, respectively, an optimal cutoff of two or more focal lesions even without underlying osteolytic lesions has been found to be of prognostic significance for progression into symptomatic disease with a 2-year rate of progression of $70-80 \%$. Positive findings of focal lesions were reported in $16 \%$ and $28 \%$ of patients, respectively $(35,36)$. This observation has led to the inclusion of MRI focal lesions as an MDE, leading to the recommendation to treat these patients (2).

For the same reasons mentioned in the MGUS section, we recommend WBCT as the first imaging technique for exclusion of osteolytic lesions. If imaging findings are inconclusive, the same imaging technique should be repeated after 3-6 months. In a study by Merz et al., increase in number or size of focal lesions in SMM patients on follow-up WBMRI studies were prognostic for progression to active MM requiring treatment compared to SMM patients who had stable FL findings (37). If only a WBMRI has been performed initially, bone imaging - i.e. WBCT - should be done to exclude lytic lesions. It has been shown that the risk of progression from SMM to MM decreases over time, reflecting the potential presence of a group of patients with high risk SMM and such with a rather MGUS-like type (38). Therefore, if no signs of progression occur, regular imaging might be reduced or stopped after 5 years, especially in patients without high risk features. Due to the complementary findings of WBCT (osteolytic lesions) and WBMRI (bone marrow lesions) in patients with only one focal lesion, an alternating approach might be considered. Figure 3 shows an algorithm for imaging in patients with SMM. 


\section{Recommendations for imaging in SMM}

- In SMM, accurate diagnosis is critical and hence CSS is not recommended to determine presence or absence of bone disease.

- WBCT is the first choice to exclude osteolytic lesions.

- If WBCT is negative, we recommend consideration of WBMRI (or MRI of spine and pelvis, if WBMRI is not available) as the next diagnostic step because of its high sensitivity and the necessity of excluding focal lesions as MDEs.

- PET-CT can be used in place of WBCT.

- PET-CT can be used in place of WBMRI if the procedure is not feasible or if there are other contraindications or patient factors that preclude its use.

- The same technique used at initial diagnosis should be repeated in yearly intervals for at least 5 years, depending on risk factors. Furthermore, alternating CT examinations should be performed in certain circumstances to exclude small osteolytic lesions

\section{Multiple myeloma}

\section{Imaging at first diagnosis}

Similar to investigations on early stages, both PET-CT and MRI have been shown to provide prognostic information based on the presence and number of focal lesions, as well as diffuse infiltration of the bone marrow. In analyses of newly diagnosed patients with MM in the context of the total therapy protocols, more than 7 focal lesions in spinal MRI and more than 3 lesions in PET-CT have been shown to be of adverse prognostic significance (39-42). These data have been confirmed in another cohort of 192 patients by the MM group in Bologna and Udine (43). Although these results are prognostic at diagnosis (46) and do serve as a reference point for emerging new lesions at progression, they are not utilized to escalate or de-escalate therapy at the moment. Furthermore, the post-therapy imaging interpretation should be done in the context of the same imaging technique (WBMRI or PET-CT) that was used at baseline assessment. The novel imaging techniques give a comprehensive assessment of total tumor mass, extramedullary disease and potentially clinically relevant impairment of the skeletal system or involved organs.

In clinical routine, a careful evaluation of the extent of bone destruction is of utmost importance. Therefore, WBCT is the first technique recommended as a minimal requirement that should be used. If this does not show any signs of lytic lesions or osteoporosis, preferably WBMRI should be performed as per the reasons mentioned in the paragraph on SMM. Due to the prognostic effect at both first diagnosis as well as after completion of therapy, PET-CT is recommended within clinical trials and also where available outside of them. In figure 4 the recommendations for imaging in patients with $\mathrm{MM}$ in clinical routine and clinical trials, respectively, are outlined. For patients who are negative in all imaging techniques but show other MDEs, the general recommendations of the IMWG should be considered.

Certain emergent clinical situations may necessitate imaging, such as an MRI to rule out cord compression or a CT to assess bone integrity or stability. 


\section{Recommendations for imaging in $\mathrm{MM}$ at first diagnosis}

- CSS is not recommended to determine presence or absence of bone disease in myeloma.

- WBCT is the first choice to assess presence and extent of osteolytic lesions.

- $\quad$ PET-CT can be used in place of WBCT.

- If WBCT is negative, and there are no other MDEs, we recommend consideration of WBMRI (or MRI of spine and pelvis, if WBMRI is not available) as the next diagnostic step because of its high sensitivity and the necessity to exclude focal lesions as MDEs.

- PET-CT can be used in place of WBMRI if the procedure is not feasible or if there are other contraindications or patient factors that preclude its use.

- In clinical trials, PET-CT is preferred to create a baseline for response assessment

\section{Imaging during follow-up or therapy for response evaluation}

Recently the IMWG has published an updated version of the criteria for assessment of treatment response in MM. Here for the first time the possibility of a focal infiltration pattern was taken into account mentioning that especially in case of a CR or even MRD negative stage, information derived from whole body imaging has to be considered (44). This is because both CR and MRD negativity are defined by low and not measurable plasma cell counts in the bone marrow, respectively. Since these examinations are usually performed in the pelvic bone where the bone marrow is best accessible, one can miss residual disease at other sites in the body. Several studies have proven that residual focal lesions in either PETCT, spinal or WBMRI are of adverse prognostic significance $(39,45-47)$. In an Italian study published by Zamagni et al., the progression-free survival was 44 versus 84 months for patients with versus without residual focal lesions (45). Figure 5 shows a potential algorithm for imaging during follow-up and for response evaluation. It has to be mentioned again that during follow up the same technique as at baseline should be used to provide comparability. Follow-up imaging does not only provide information on progression of the disease. Instead, the more widespread use of computed tomography has revealed that lytic lesions can show signs of bone healing, and residual lesions immediately after intensive therapy can become negative after a longer follow-up. As a caveat, it has to be mentioned that in PET-CT reparative osteogenesis in rare cases can lead to false positive results. Therefore, in case of PET-avid focal lesions in the setting of serological response, these should be compared to the CT part of the examination which would show sclerotic versus further lytic activity.

\section{Recommendations for imaging in MM during follow up and therapy}

Depending on availability of baseline examinations and initial results either WBCT to provide a baseline bone status for potential relapse or a PET-CT as part of response assessment should be performed. In patients with residual lesions in PET-CT, yearly follow up is recommended because these patients have a high risk of an early progression

\section{Imaging in relapsed multiple myeloma}

At relapse, MRI has been proven to be of value for early detection of recurrent bone marrow infiltration with a slight superiority over PET-CT, while PET-CT was better in early detection of response to salvage therapy (48). However, these findings too have not been incorporated to change therapy while managing relapsed MM patients. We recommend WBCT when a relapse is suspected (e.g. serological relapse or progression) to assess the extent and dynamic of bone destruction as the most clinically relevant parameter. These 
recommendations are summarized in figure 6.

\section{How to perform and report bone marrow findings in PET-CT and WBMRI}

Some studies investigating MRI in monoclonal plasma cell disorders only included the spine and the pelvis and sometimes the skull (axial MRI). A comparative study of axial and WBMRI revealed that 10 of 100 patients had focal lesions only in the extra-axial skeleton which would have been missed if only a spinal MRI had been performed (49). Therefore, if available WBMRI should be the preferred imaging technique.

It would be beyond the scope of this manuscript to describe in detail which specifications for WBMRI and PET-CT should be used. However, recommendations for the interpretation of PET-CT have been published by the IMWG and are currently refined by the same group (50). In brief, focal lesions in PET-CT at first diagnosis have been defined by a tracer uptake higher than that of hematopoietic bone marrow and/or that of the liver. A diffuse uptake should be reported if it lies above that of the liver.

Recommendations for technical specifications of WBMRI as well as WBCT by interdisciplinary groups of radiologists, physicists, and hematologists have been accepted for publication (18)(Messiou et al, accepted, Radiology 2018). MRI focal lesions are characterized by hypointensity in T1- and corresponding hyperintensity in T2-weighted or inversion recovery images. Diffuse infiltration can be identified if the signal intensity is decreased homogeneously in T1- and increased in T2-weighted images. As a reference, the signal intensity of the intervertebral disk should be used.

All whole body imaging should include the vertex of the skull and at least the knees. If protocols are available, the lower extremities should be displayed in full.

Based on the generally used slice thickness of $5 \mathrm{~mm}$ of the WBMRI, a minimum diameter of a focal lesion to be defined as such was arbitrarily set at $5 \mathrm{~mm}$. From a hematologist's perspective the following information should be provided when reporting on PET-CT or WBMRI. 
Recommendations

First diagnosis

A radiological report on whole body imaging in patients with monoclonal plasma cell disorders should include information on the following:

1) Infiltration/ bone destruction pattern

a. Minimal (normal appearing)

b. Focal lesions

c. Diffuse Infiltration/ bone destruction

d. Mixed (focal lesions on diffuse background)

2) Absolute number of focal lesions

a. For WBMRI 0, 1, 2-7, >7

b. For PET-CT $0,1-3,>3$

3) Number of fractures (new versus old, location, likelihood of malignant versus benign cause)

4) Extramedullary disease

5) Soft tissue masses growing out of the bone marrow into the surrounding tissue

6) Infiltration of the long bones

7) Evidence of surgical procedures at the skeletal system

8) Incidental findings

\section{In Remission}

A 5-stage scoring system is recommended differentiating the following findings regarding response to therapy in imaging (guidelines papers for WBCT, WBMRI and PET-CT):

1) Response

a. Normalization of bone marrow signal in previously affected areas

b. Decrease in number and/or size of focal lesions

c. Resolution of severely infiltrated bone marrow infiltrate into focal lesions

d. Decrease of number and/or size of soft tissue tumors (para- and

2) No change extramedullary)

3) Progression
a. Worsening of diffuse bone marrow signal or new appearance of infiltration in previously unaffected areas
b. Increase in number and/or size of focal lesions
c. Merging of focal lesions into severely infiltrated bone marrow
d. Increasing in size and/or number of soft tissue tumors (para- and extramedullary)

Specifics for MRI

Cystic/ liquid transformation of focal lesions after therapy

This manuscript aims to provide guidelines for how to use current imaging modalities. New PET-tracers and novel technologies such as double energy CT will likely be introduced into clinical routine in the not too distant future. This might lead to a necessity for changes in the current recommendations.

\section{ACKNOWLEDGEMENT}

Tim Weber (assessment of CT), Christina Messiou (assessment of MRI), Benjamin McCarthy for correcting linguistig mistakes in the article. 


\section{REFERENCES}

1. Landgren O, Kyle RA, Pfeiffer RM, Katzmann JA, Caporaso NE, Hayes RB, et al. Monoclonal gammopathy of undetermined significance (MGUS) consistently precedes multiple myeloma: a prospective study. Blood. 2009 May 28;113(22):5412-7.

2. Rajkumar SV, Dimopoulos MA, Palumbo A, Blade J, Merlini G, Mateos M-V, et al. International Myeloma Working Group updated criteria for the diagnosis of multiple myeloma. Lancet Oncol. 2014 Nov;15(12):e538-548.

3. Ludwig H, Miguel JS, Dimopoulos MA, Palumbo A, Garcia Sanz R, Powles R, et al. International Myeloma Working Group recommendations for global myeloma care. Leukemia. 2014 May;28(5):981-92.

4. Kyle RA, Gertz MA, Witzig TE, Lust JA, Lacy MQ, Dispenzieri A, et al. Review of 1027 patients with newly diagnosed multiple myeloma. Mayo Clin Proc. 2003 Jan;78(1):2133.

5. Hillengass J, Landgren O. Challenges and opportunities of novel imaging techniques in monoclonal plasma cell disorders: imaging "early myeloma." Leuk Lymphoma. 2013 Jul;54(7):1355-63.

6. Dimopoulos M, Terpos E, Comenzo RL, Tosi P, Beksac M, Sezer O, et al. International myeloma working group consensus statement and guidelines regarding the current role of imaging techniques in the diagnosis and monitoring of multiple Myeloma. Leukemia. 2009 Sep;23(9):1545-56.

7. International Myeloma Working Group. Criteria for the classification of monoclonal gammopathies, multiple myeloma and related disorders: a report of the International Myeloma Working Group. Br J Haematol. 2003 Jun;121(5):749-57.

8. Edelstyn GA, Gillespie PJ, Grebbell FS. The radiological demonstration of osseous metastases. Experimental observations. Clin Radiol. 1967 Apr;18(2):158-62.

9. Hillengass J, Moulopoulos LA, Delorme S, Koutoulidis V, Hielscher T, Engelhart J, et al. Findings of Whole Body Computed Tomography Compared to Conventional Skeletal Survey in Patients with Monoclonal Plasma Cell Disorders - a Study of the International Myeloma Working Group. Blood. 2016 Dec 2;128(22):4468-4468.

10. Hinge M, Andersen KT, Lund T, Jørgensen HB, Holdgaard PC, Ormstrup TE, et al. Baseline bone involvement in multiple myeloma - a prospective comparison of conventional $\mathrm{X}$-ray, low-dose computed tomography, and 18flourodeoxyglucose positron emission tomography in previously untreated patients. Haematologica. 2016 Oct;101(10):e415-8. 11. Kröpil P, Fenk R, Fritz LB, Blondin D, Kobbe G, Mödder U, et al. Comparison of whole-body 64-slice multidetector computed tomography and conventional radiography in staging of multiple myeloma. Eur Radiol. 2008 Jan;18(1):51-8.

12. Wolf MB, Murray F, Kilk K, Hillengass J, Delorme S, Heiss C, et al. Sensitivity of whole-body CT and MRI versus projection radiography in the detection of osteolyses in patients with monoclonal plasma cell disease. Eur J Radiol. 2014 Jul;83(7):1222-30.

13. Nishida Y, Matsue Y, Suehara Y, Fukumoto K, Fujisawa M, Takeuchi M, et al. Clinical and prognostic significance of bone marrow abnormalities in the appendicular skeleton detected by low-dose whole-body multidetector computed tomography in patients with multiple myeloma. Blood Cancer J. 2015 Jul 31;5:e329.

14. Horger M, Kanz L, Denecke B, Vonthein R, Pereira P, Claussen CD, et al. The benefit of using whole-body, low-dose, nonenhanced, multidetector computed tomography for follow-up and therapy response monitoring in patients with multiple myeloma. Cancer. 2007 Apr 15;109(8):1617-26.

15. Horger M, Claussen CD, Bross-Bach U, Vonthein R, Trabold T, Heuschmid M, et al. Whole-body low-dose multidetector row-CT in the diagnosis of multiple myeloma: an 
alternative to conventional radiography. Eur J Radiol. 2005 May;54(2):289-97. 16. Ippolito D, Besostri V, Bonaffini PA, Rossini F, Di Lelio A, Sironi S. Diagnostic value of whole-body low-dose computed tomography (WBLDCT) in bone lesions detection in patients with multiple myeloma (MM). Eur J Radiol. 2013 Dec;82(12):2322-7.

17. Cretti F, Perugini G. Patient dose evaluation for the whole-body low-dose multidetector CT (WBLDMDCT) skeleton study in multiple myeloma (MM). Radiol Med (Torino). 2016 Feb;121(2):93-105.

18. Moulopoulos LA, Koutoulidis V, Hillengass J, Zamagni E, Aquerreta JD, Roche CL, et al. Recommendations for acquisition, interpretation and reporting of whole body low dose CT in patients with multiple myeloma and other plasma cell disorders: a report of the IMWG Bone Working Group. Blood Cancer J. 2018 Oct 4;8(10):95.

19. Rasche L, Chavan SS, Stephens OW, Patel PH, Tytarenko R, Ashby C, et al. Spatial genomic heterogeneity in multiple myeloma revealed by multi-region sequencing. Nat Commun. 2017 16;8(1):268.

20. Hillengass, J, Ellert, E., Spira, D., Hemmer, S., Wagner, B., Andrulis, M., et al. Comparison of plasma cell infiltration in random samples of the bone marrow and osteolyses acquired by CT-guided biopsy in patients with symptomatic multiple myeloma. In: Journal of Clinical Oncology [Internet]. 2016 [cited 2018 Feb 14]. p. Suppl; abstr 8040. Available from: https://meetinglibrary.asco.org/record/126334/abstract

21. Grobner T. Gadolinium--a specific trigger for the development of nephrogenic fibrosing dermopathy and nephrogenic systemic fibrosis? Nephrol Dial Transplant Off Publ Eur Dial Transpl Assoc - Eur Ren Assoc. 2006 Apr;21(4):1104-8.

22. Koh D-M, Collins DJ. Diffusion-weighted MRI in the body: applications and challenges in oncology. AJR Am J Roentgenol. 2007 Jun;188(6):1622-35.

23. Sachpekidis C, Hillengass J, Goldschmidt H, Mosebach J, Pan L, Schlemmer H-P, et al. Comparison of (18)F-FDG PET/CT and PET/MRI in patients with multiple myeloma. Am J Nucl Med Mol Imaging. 2015;5(5):469-78.

24. Caserta E, Chea J, Minnix M, Viola D, Vonderfecht S, Yazaki P, et al. Copper 64labeled daratumumab as a PET/CT imaging tracer for multiple myeloma. Blood. $2018 \mathrm{Feb}$ 15;131(7):741-5.

25. Kyle RA, Therneau TM, Rajkumar SV, Larson DR, Plevak MF, Offord JR, et al. Prevalence of monoclonal gammopathy of undetermined significance. N Engl J Med. 2006 Mar 30;354(13):1362-9.

26. Kyle RA, Larson DR, Therneau TM, Dispenzieri A, Kumar S, Cerhan JR, et al. LongTerm Follow-up of Monoclonal Gammopathy of Undetermined Significance. N Engl J Med. 2018 18;378(3):241-9.

27. Hillengass J, Weber M-A, Kilk K, Listl K, Wagner-Gund B, Hillengass M, et al. Prognostic significance of whole-body MRI in patients with monoclonal gammopathy of undetermined significance. Leukemia. 2014 Jan;28(1):174-8.

28. Bhutani M, Turkbey B, Tan E, Korde N, Kwok M, Manasanch EE, et al. Bone marrow abnormalities and early bone lesions in multiple myeloma and its precursor disease: a prospective study using functional and morphologic imaging. Leuk Lymphoma. 2016 May;57(5):1114-21.

29. Minarik J, Krhovska P, Hrbek J, Pika T, Bacovsky J, Herman M, et al. Prospective comparison of conventional radiography, low-dose computed tomography and magnetic resonance imaging in monoclonal gammopathies. Biomed Pap Med Fac Univ Palacky Olomouc Czechoslov. 2016 Jun;160(2):305-9.

30. Yuh WT, Zachar CK, Barloon TJ, Sato Y, Sickels WJ, Hawes DR. Vertebral compression fractures: distinction between benign and malignant causes with MR imaging. Radiology. 1989 Jul;172(1):215-8. 
31. Baker LL, Goodman SB, Perkash I, Lane B, Enzmann DR. Benign versus pathologic compression fractures of vertebral bodies: assessment with conventional spin-echo, chemicalshift, and STIR MR imaging. Radiology. 1990 Feb;174(2):495-502.

32. Baur A, Stäbler A, Brüning R, Bartl R, Krödel A, Reiser M, et al. Diffusion-weighted MR imaging of bone marrow: differentiation of benign versus pathologic compression fractures. Radiology. 1998 May;207(2):349-56.

33. Nahi H, Genell A, Wålinder G, Uttervall K, Juliusson G, Karin F, et al. Incidence, characteristics, and outcome of solitary plasmacytoma and plasma cell leukemia. Populationbased data from the Swedish Myeloma Register. Eur J Haematol. 2017 Sep;99(3):216-22.

34. Paiva B, Chandia M, Vidriales M-B, Colado E, Caballero-Velázquez T, Escalante F, et al. Multiparameter flow cytometry for staging of solitary bone plasmacytoma: new criteria for risk of progression to myeloma. Blood. 2014 Aug 21;124(8):1300-3.

35. Kastritis E, Moulopoulos LA, Terpos E, Koutoulidis V, Dimopoulos MA. The prognostic importance of the presence of more than one focal lesion in spine MRI of patients with asymptomatic (smoldering) multiple myeloma. Leukemia. 2014 Dec;28(12):2402-3. 36. Hillengass J, Fechtner K, Weber M-A, Bäuerle T, Ayyaz S, Heiss C, et al. Prognostic significance of focal lesions in whole-body magnetic resonance imaging in patients with asymptomatic multiple myeloma. J Clin Oncol Off J Am Soc Clin Oncol. 2010 Mar 20;28(9):1606-10.

37. Merz M, Hielscher T, Wagner B, Sauer S, Shah S, Raab MS, et al. Predictive value of longitudinal whole-body magnetic resonance imaging in patients with smoldering multiple myeloma. Leukemia. 2014 Sep;28(9):1902-8.

38. Kyle RA, Remstein ED, Therneau TM, Dispenzieri A, Kurtin PJ, Hodnefield JM, et al. Clinical course and prognosis of smoldering (asymptomatic) multiple myeloma. N Engl J Med. 2007 Jun 21;356(25):2582-90.

39. Bartel TB, Haessler J, Brown TLY, Shaughnessy JD, van Rhee F, Anaissie E, et al. F18-fluorodeoxyglucose positron emission tomography in the context of other imaging techniques and prognostic factors in multiple myeloma. Blood. 2009 Sep 3;114(10):2068-76. 40. Walker R, Barlogie B, Haessler J, Tricot G, Anaissie E, Shaughnessy JD, et al. Magnetic resonance imaging in multiple myeloma: diagnostic and clinical implications. $\mathrm{J}$ Clin Oncol Off J Am Soc Clin Oncol. 2007 Mar 20;25(9):1121-8.

41. Usmani SZ, Mitchell A, Waheed S, Crowley J, Hoering A, Petty N, et al. Prognostic implications of serial 18-fluoro-deoxyglucose emission tomography in multiple myeloma treated with total therapy 3. Blood. 2013 Mar 7;121(10):1819-23.

42. Davies FE, Rosenthal A, Rasche L, Petty NM, McDonald JE, Ntambi JA, et al. Treatment to suppression of focal lesions on positron emission tomography-computed tomography is a therapeutic goal in newly diagnosed multiple myeloma. Haematologica. 2018 Jun;103(6):1047-53.

43. Zamagni E, Patriarca F, Nanni C, Zannetti B, Englaro E, Pezzi A, et al. Prognostic relevance of 18-F FDG PET/CT in newly diagnosed multiple myeloma patients treated with up-front autologous transplantation. Blood. 2011 Dec 1;118(23):5989-95.

44. Kumar S, Paiva B, Anderson KC, Durie B, Landgren O, Moreau P, et al. International Myeloma Working Group consensus criteria for response and minimal residual disease assessment in multiple myeloma. Lancet Oncol. 2016 Aug;17(8):e328-346.

45. Zamagni E, Nanni C, Mancuso K, Tacchetti P, Pezzi A, Pantani L, et al. PET/CT Improves the Definition of Complete Response and Allows to Detect Otherwise Unidentifiable Skeletal Progression in Multiple Myeloma. Clin Cancer Res Off J Am Assoc Cancer Res. 2015 Oct 1;21(19):4384-90.

46. Moreau P, Attal M, Caillot D, Macro M, Karlin L, Garderet L, et al. Prospective Evaluation of Magnetic Resonance Imaging and [18F]Fluorodeoxyglucose Positron Emission 
Tomography-Computed Tomography at Diagnosis and Before Maintenance Therapy in Symptomatic Patients With Multiple Myeloma Included in the IFM/DFCI 2009 Trial: Results of the IMAJEM Study. J Clin Oncol Off J Am Soc Clin Oncol. 2017 Sep 1;35(25):2911-8. 47. Hillengass J, Ayyaz S, Kilk K, Weber M-A, Hielscher T, Shah R, et al. Changes in magnetic resonance imaging before and after autologous stem cell transplantation correlate with response and survival in multiple myeloma. Haematologica. 2012 Nov;97(11):1757-60. 48. Spinnato P, Bazzocchi A, Brioli A, Nanni C, Zamagni E, Albisinni U, et al. Contrast enhanced MRI and ${ }^{18}$ F-FDG PET-CT in the assessment of multiple myeloma: a comparison of results in different phases of the disease. Eur J Radiol. 2012 Dec;81(12):4013-8.

49. Bäuerle T, Hillengass J, Fechtner K, Zechmann CM, Grenacher L, Moehler TM, et al. Multiple myeloma and monoclonal gammopathy of undetermined significance: importance of whole-body versus spinal MR imaging. Radiology. 2009 Aug;252(2):477-85.

50. Cavo M, Terpos E, Nanni C, Moreau P, Lentzsch S, Zweegman S, et al. Role of (18)F-FDG PET/CT in the diagnosis and management of multiple myeloma and other plasma cell disorders: a consensus statement by the International Myeloma Working Group. Lancet Oncol. 2017 Apr;18(4):e206-17.

\section{FIGURE LEGENDS}

Figure 1: Imaging algorithm for patients with non-IgM MGUS

Figure 2: Imaging algorithm for patients with SPC

Figure 3: Imaging algorithm for patients with SMM

Figure 4: Imaging algorithm for patients with MM at first diagnosis

Figure 5: Imaging during follow-up therapy for response evaluation

Figure 6: Imaging algorithm for patients with suspicion of relapsed/progressing MM 